\title{
Incontrare un Altro Mondo: Immagin, InTERCUltura e PartecipaZione nella Classe di Italiano come Lingua Straniera
}

\author{
Simona Bartoli Kucher*
}

ABSTRACT: L'articolo vuole mostrare - tra riflessioni teoriche e pratica didattica - come il film e il romanzo multimodale possano avere un ruolo fondamentale in: un approccio didattico al cui centro stanno gli apprendenti in qualità di attanti culturali. Se testi e film in lingua straniera presentano contenuti legati al loro mondo, se raccontano storie che li coinvolgono dal punto di vista emozionale e cognitivo, contribuiscono a potenziare la competenza testuale, la competenza narrativa e la competenza interculturale in lingua straniera, dando un importante contributo educativo e di sviluppo della personalità.

PAROLE CHIAVE: testo e cultura visiva; intercultura; partecipazione; romanzo multimodale.

RESUMO: O presente artigo pretende mostrar - entre reflexões teóricas e prática didática - como o filme e o romance multimodal podem ter um papel fundamental em uma abordagem didática em cujo centro estão os aprendizes como actantes culturais. Se textos e filmes em língua estrangeira apresentam conteúdos ligados a seu mundo, se narram histórias que os envolvem do ponto de vista emocional e cognitivo, contribuem para potencializar a competência textual, a competência narrativa e a competência intercultural em língua estrangeira, dando uma

*Docente di lingua, cultura e didattica dell'italiano come lingua straniera all'Istituto di Romanistica della Karl-Franzens Universität Graz (Austria). 
importante contribuição educacional e ligada ao desenvolvimento da personalidade. PALAVRAS-CHAVE: texto e cultura visual; intercultura; participação; romance multimodal.

ABSTRACT: The following paper aims to point out - through theoretical reflection and didactic practice - how films and multimodal novels can assume an important role in a didactic approach which focalizes on the learners as active cultural subjects. If texts and films in a foreign language present contents related to aspects and contexts of their own experience and cultural knowledge, if they tell stories that involve students cognitively and emotionally, they will contribute to heightening the narrative, textual and intercultural competences, finally contributing to their education and formation of their personality.

KEYWORDS: text and visual culture; interculture; participation; multimodal novel. 


\section{Introduzione}

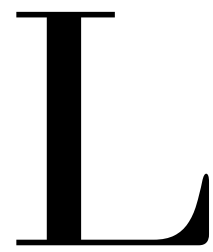

e riflessioni che qui verranno presentate si basano sull'idea di attivare nell'insegnamento delle lingue straniere un'ottica interculturale, sviluppando negli apprendenti un atteggiamento partecipativo.

Che ruolo deve e può svolgere l'intercultura nella didattica e nell'insegnamento di una lingua straniera ${ }^{1}$, nel nostro caso dell'italiano? A questa domanda si cercherà di rispondere presentando un'esperienza di collaborazione tra l'Istituto di Italianistica dell'Università di Graz ${ }^{2}$ e alcune

$1 \mathrm{Si}$ tenga presenta che da quando è stato redatto il Quadro Comune Europeo di Riferimento per le Lingue (da ora in poi qui QCER), l'insegnamento delle lingue moderne e di conseguenza i piani di studio relativi alle lingue moderne nelle scuole e nell'università si orientano alle competenze. La competenza interculturale viene citata nel QCER in modo astratto, per cui non vengono usati descrittori né indicati criteri didattici operativi.

2 Si tratta di un progetto di collaborazione tra università e scuole, iniziato nel 2007 dall'università allo scopo di collegare la ricerca pedagogica sul mondo della scuola e la ricerca didattica dell’Università Karl Franzens con la prassi delle scuole della Stiria (regione meridionale dell'Austria di cui Graz è il capoluogo). Il progetto cui si farà riferimento, partito nel 2013 e coordinato da chi scrive, porta il titolo "CAPIRE STORIE - RACCONTARE STORIE. Insegnare e imparare la competenza narrativa e la competenza interculturale con testi letterari e film". 
classi di un liceo della città in cui l'italiano viene studiato come seconda lingua straniera ${ }^{3}$. Nell'ambito del progetto ${ }^{4}$ agli studenti universitari e agli studenti liceali sono stati presentati il film di Silvio Muccino Un altro mondo ${ }^{5}$ e il romanzo di Carlo Lucarelli Febbre gialla (1997) allo scopo di attivare la loro partecipazione culturale e sociale sulla base del potenziamento della competenza linguistico comunicativa.

Uno dei punti di partenza del lavoro è stato il valore che le immagini possono offrire alla glottodidattica. In una società multimediale in cui le informazioni circolano attraverso immagini e multiple media formats più che attraverso fonti stampate ${ }^{6}$, anche nell'insegnamento delle lingue straniere si dovrebbe approfondire da un lato il potenziale rappresentato dalla competenza visiva - visto che le immagini aiutano la comprensione e favoriscono la memorizzazione (ISSING, HANNEMANN, 1983) -, tenendo conto dall'altro del forte influsso che le emozioni forti (come quelle veicolate dai film) hanno sui processi di fissazione della memoria (FABBRO, 1996, p. 101; ARNOLD, 2002, p. 14). Della necessità di colmare questi deficit nella didattica delle lingue straniere si parlerà nei paragrafi che seguono.

\section{Un'immagine vale più di mille parole}

Gli studenti liceali e gli studenti universitari sono stati confrontati, per cominciare, con screenshots tratti dalle prime sequenze del film di S. Muccino Un altro mondo (2010), nell'intento di invitarli a collaborare colmando gli spazi vuoti ${ }^{7}(\mathrm{ECO}, 1994)$. Agli studenti è stato proposto di raccontare oralmente una storia, prestando alle immagini i propri sentimenti e la propria forza immaginativa (SARTRE, 1964; ECO, 2002).

3 Il sistema scolastico austriaco prevede, dopo 4 anni di scuola elementare, 8 anni di scuola superiore in cui l'inglese viene insegnato come prima lingua straniera obbligatoria. Sono molti i licei e gli istituti superiori che prevedono, a partire dal terzo o dal quinto anno, l'insegnamento di una seconda lingua straniera (a scelta tra francese, italiano, spagnolo e latino. Nelle regioni dell'Austria meridionale - Stiria e Carinzia - l'italiano occupa il primo posto).

4 Il lavoro è stato realizzato, nelle classi liceali, dall'insegnante di italiano coadiuvata da un'insegnante di italiano in formazione che aveva seguito all'università i corsi specifici di didattica della letteratura di lingua straniera e di didattica del film.

5 Silvio Muccino, Un altro mondo, 2010, con Silvio Muccino, Isabella Ragonese, Michael Rainey Jr, Maya Sansa, Italia, 109'. Il film è la trasposizione cinematografica del romanzo omonimo di Carla Vangelista: C. Vangelista, Un altro mondo, Milano: Feltrinelli, 2009.

6 Si veda lo statement del NCSS (National Council for Social Studies) sui Media Literacy, in cui si legge: "We live in a multimedia age where the majority of information people receive comes less often from print sources and more typically from highly constructed visual images, complex sound arrangements, and multiple media formats". In: http:// www.socialstudies.org/positions/medialiteracy (ultimo accesso 7.7.2014).

$7 \mathrm{Il}$ testo vive in quanto c'è un lettore che lo rende comunicativo: "ogni testo è una macchina pigra che chiede al lettore di fare parte del proprio lavoro" (ECO, 1994, p. 3). 

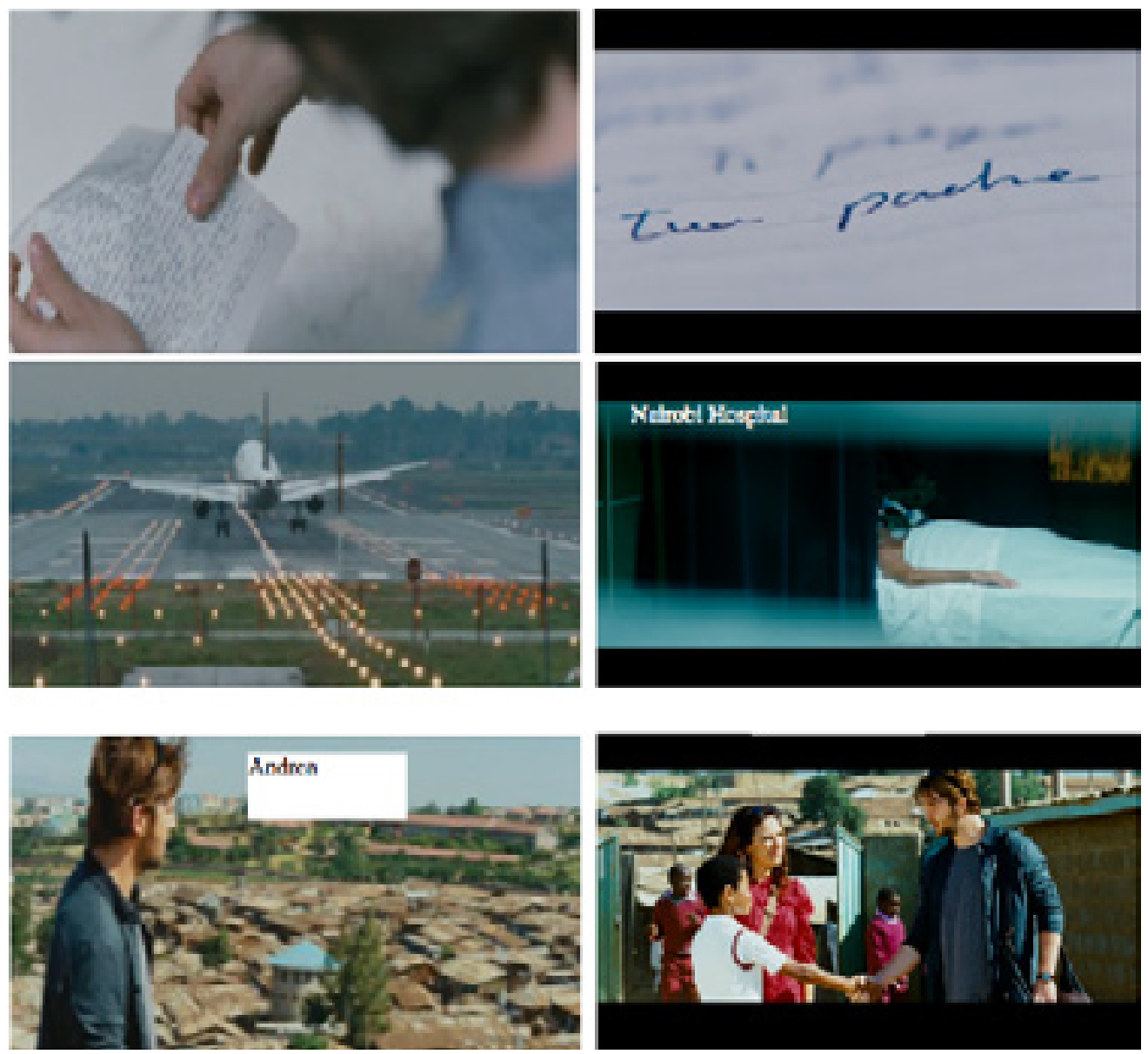

Sia gli studenti liceali che quelli universitari avevano alle spalle alcuni anni di studio della lingua (i primi avevano già studiato italiano per tre anni, gli altri partivano dal livello linguistico comunicativo $\mathrm{B} 1^{8}$ ), quindi disponevano di tutta una serie di strategie linguistiche e naturalmente di una conoscenza del mondo legata alla rispettiva età e alla rispettiva acculturazione. Chiedere agli studenti di leggere le immagini, di riempire gli spazi vuoti, di raccontare una storia a partire dalle immagini proposte, chiama in causa la loro expectancy grammar, quel processo

8 Per iniziare il curriculum universitario all'Istituto di Romanistica dell'Università di Graz bisogna dimostrare in un test d'ingresso di aver raggiunto il livello A2. Le attività didattiche a cui si sta facendo riferimento sono state proposte a studenti del secondo semestre. 
che governa ogni forma di previsione veicolata attraverso un atto linguistico. "La grammatica che governa il processo di previsione" (BALBONI, 2002, p. 111) ha che fare con:

- l'immaginare quel che potrebbe essere accaduto attivando la competenza narrativa e la competenza discorsiva (raccontare usando in modo coerente il lessico, le strutture della lingua, le frasi idiomatiche, la coesione testuale);

- $\quad$ il fare ricorso al lessico (noto o nuovo) relativo alle situazioni individuate, a partire dal patrimonio lessicale già in possesso degli apprendenti;

- la competenza culturale a partire dal patrimonio culturale già in possesso degli apprendenti e dalle loro esperienze individuali di socializzazione.

Gli apprendenti di una lingua straniera danno significato alle immagini con l'aiuto della loro enciclopedia e delle esperienze già in loro possesso in modo da sviluppare un quadro coerente della situazione presa in esame e dei personaggi che ne fanno parte.

\subsection{Tra immagini e parole: raccontare un altro mondo}

Si tratta di un approccio didattico in cui le immagini costituiscono il punto di partenza per ricostruire e raccontare una storia. La storia dell'incontro tra un giovane romano viziato e vizioso e di un bambino di colore cresciuto in un altro mondo, in un mondo e una cultura completamente diversi, consente un discorso culturale orientato alle esperienze sociali degli studenti. Lavorare con immagini come quelle da cui siamo partiti significa attivare capacità comunicative preparando gli apprendenti all'interazione sociale, a dimostrare di "saper vivere in modo autonomo nella società e partecipare a importanti processi sociali” (HALLET, 2007, p. 31$)^{9}$.

Un compito come quello proposto, orientato all'azione e alla produzione - che non prevede risposte e/o proposte sbagliate perché ogni proposta di lettura è giusta -, mette alla prova l'abilità discorsiva in lingua straniera, oltre che la capacità di partecipazione offrendo ad ogni apprendente una motivante occasione di produzione orale o di produzione scritta oltre che di competenza (inter)culturale nella lingua oggetto di studio. Fondamentale risulta la contestualizzazione semiotica di una situazione piena di richiami interculturali in cui gli apprendenti giocano il

9, ,[...], dass sie auf selbstbestimmte Weise in einer Gesellschaft leben und an wichtigen gesellschaflichen Prozessen partizipieren können ". Nel testo è stata riportata la traduzione italiana di Simona Bartoli Kucher. In assenza di traduzione pubblicata, si riporterà la traduzione di chi scrive. 
ruolo di attanti culturali che

partecipano sempre anche a processi sociali reali. [...] Le studentesse e gli studenti prendono parte al processo di apprendimento e di insegnamento come soggetti culturali con le loro idee relative a valori, con i loro atteggiamenti, con le loro opinioni e le loro costruzioni di senso (HALLET 2012, p. 11-12) ${ }^{10}$.

\section{Una competenza complessa: tra abilità discorsiva e sensibilità interculturale}

Passare alla visione delle sequenze del film ${ }^{11}$ in cui compaiono le immagini catturate dalle schermate consente agli apprendenti di verificare le prime ipotesi: un'attività didattica che offre un'ulteriore occasione di produzione orale, oltre che la possibilità di introdurre quell'alfabeto del cinema che rappresenta ancora un deficit nella didattica delle lingue straniere moderne. I primi 8 minuti del film introducono, attraverso immagini e musica, nella vita sregolata del protagonista e dei suoi amici, con brevi flashback sulla sua infanzia. Nella lunga introduzione, realizzata con un montaggio veloce e accompagnata dalla voce fuori campo del protagonista, e nelle prime tre tracce del film, le immagini di partenza si ritrovano in cinque scene caratterizzate da unità spazio temporale:

1. (7:49). Andrea, il protagonista della storia - introdotta dalla sua voce fuori campo diegetica - superficiale e viziato, abituato a una vita dissoluta, mantenuto ai piani alti della società romana dagli assegni in bianco della madre, nel giorno del suo ventottesimo compleanno riceve inaspettatamente da Nairobi una lettera del padre, malato terminale, che vorrebbe rivederlo;

2. (9:50-11:00). Per noia e curiosità nei confronti di una figura scomparsa dalla sua vita venti anni prima, Andrea parte da Roma alla volta della capitale keniota; 3. (12:58-14:55). All'Ospedale di Nairobi il ragazzo trova il padre sul letto di morte. Una volontaria italiana lo informa che l'uomo ha avuto un secondo figlio da una donna del posto, deceduta dopo la nascita del bambino.

10 “[...] immer an realen gesellschaftlichen Prozessen partizipieren. [...] Die Schülerinnen und Schüler nehmen als kulturelle Subjekte mit ihren eigenen Wertvorstellungen, Haltungen, Meinungen, Sinnstiftungen usw am Lern- und Unterrichtsprozess teil".

$11 \mathrm{Il} \mathrm{film} \mathrm{è} \mathrm{stato} \mathrm{un} \mathrm{flop} \mathrm{al} \mathrm{botteghino} \mathrm{ed} \mathrm{è} \mathrm{stato} \mathrm{accolto} \mathrm{con} \mathrm{molte} \mathrm{riserve} \mathrm{dalla} \mathrm{critica.} \mathrm{Si} \mathrm{veda} \mathrm{l'articolo} \mathrm{pubblicato}$ il 28 dicembre 2010 sul Sole 24 ore, che, pur riconoscendo al film un cast eccellente, un buon montaggio e ottime fotografie, lo considera mal riuscito. Si veda: http://cinema.ilsole24ore.com/film-brevi/2010-12-23/cose-dellaltromondo-00017792.php? uuid=AYJgS8tC (ultima consultazione 15.7.2014). Anche la critica della Stampa del 21.12.2010 (http://www.film.it/recensione/art/un-altro-mondo-la-nostra-recensione-7935/ ultimo accesso 15.7.2014) salva solo il personaggio di Charlie e il tentativo di "svecchiare l'immagine classica della famiglia del Mulino Bianco" affrontando temi come l'integrazione e il razzismo. 
4.(15:00-17:00). Andrea scopre di avere un fratellino di colore di 8 anni, di cui il padre lo ha nominato tutore legale. Se non si prendesse cura di lui, Sara (questo il nome della volontaria, collaboratrice del padre in un progetto di cooperazione), lo farebbe arrestare per abbandono di minore.

5. (17:28-19:31). Andrea incontra Charlie in un villaggio alle porte di Nairobi in cui Sara insegna alle donne locali a tingere e cucire vestiti perché la dignità significa anche "non vivere dei pacchi dono".

Il processo didattico mette in gioco in primo luogo la competenza ricettiva: non solo la comprensione di quanto visto e di quanto ascoltato, ma anche la comprensione di aspetti paralinguistici come la mimica, i gesti, il linguaggio del corpo, l'intonazione, i diversi registri e le varietà sociolinguistiche dell'italiano (DIADORI, 2010).

Un rapporto interrotto padre-figlio; le responsabilità dei padri nei confronti dei figli; il rapporto tra fratelli; la differenza tra due mondi e due culture diverse; la possibilità di cambiare perché "le cose non cambiano mai, ma cambiamo noi"12. Sono queste le tematiche emerse dopo la verifica della comprensione globale e analitica, operata con le tecniche didattiche consuete (clozes, ricomposizione di frasi spezzate, incastro tra parole o frasi, ricomposizione di dialoghi in cui le battute sono state mescolate, ricostruzione della battute a partire dalla visione delle sequenze senza sonoro, domande aperte o domande con risposta a scelta multipla) (BALBONI, 2013).

Per quanto biasimato dalla critica e invece sopravvalutato da produttori e regista ${ }^{13}$ e punito dal botteghino, il film - con un cast eccellente, con una colonna sonora ${ }^{14} \mathrm{e}$ un montaggio ricercati realizzati da tecnici di risaputa competenza - è stato recepito positivamente dagli studenti, che ne hanno visto e apprezzato inizialmente singole sequenze, confrontandosi poi alla fine con

12 Questa la frase pronunciata dalla volontaria nel momento in cui Andrea sembra rifiutare la responsabilità affidategli dal padre. La frase è riportata sul cover del dvd.

13 Silvio Muccino, nel 2010, con Un altro mondo al suo secondo film da regista e attore protagonista, che ha curato anche la sceneggiatura insieme a Carla Vangelista, autrice del romanzo omonimo, avrebbe definito Un altro mondo come un film "about a boy ai tempi di Obama"; secondo i produttori Un altro mondo sarebbe un film di Natale. "Con la sceneggiatura siamo rimasti fedeli al romanzo per la prima parte, che è simile a un road-movie, mentre abbiamo rivoluzionato la seconda. Non sarà un affresco sui giovani, ma un film che scava dentro il percorso interiore del protagonista senza rinunciare a qualche tono da commedia”. Si veda S. Muccino nel blog di Carla Vangelista: $w w w$. carlavangelista.it/(ultimo accesso 10.7.2014). Si veda anche la recensione di Boris Sollazzo sul Sole 24 ore del 28 dicembre 2010: http://www.ilsole24ore.com/art/cultura/2010-12-28/silvio-muccino-cose-altro-130254.shtml\# (ultimo accesso 16.7.2014).

14 Per la colonna sonora Muccino ha utilizzato il brano Secret Garden di Bruce Springsteen, Hanno ucciso l'Uomo ragno degli 883 e I Watussi di Edoardo Vianello. 
l'intera pellicola.

L'esperienza della visione in classe ha evidenziato che le sequenze prima indicate del film presentano situazioni complesse, simili a quelle della realtà, che hanno creato l'occasione di parlare della realtà, di discutere di problematiche analoghe. Come si reagisce di fronte alla presenza del "diverso", alla sua irruzione nel nostro mondo? Lo straniero suscita paura o fascino? Lo straniero rappresenta un arricchimento o una minaccia? In ogni caso l'incontro tra Andrea e Charlie richiede la soluzione di un problema simile a quelli della realtà in cui (anche) la scuola è multiculturale e plurilingue, spingendo gli spettatori apprendenti - alla luce di un mondo fittizio - a riflettere sul proprio modo di pensare e di agire (HALLET; NÜNNING, 2009) ${ }^{15}$.

Assegnare agli apprendenti il compito di raccontare e/o mettere in scena le sequenze viste e ascoltate 1. dal punto di vista del protagonista ricco e viziato, 2. dal punto di vista della volontaria, 3. dal punto di vista del bambino orfano proveniente da un' altra cultura, proponendo una continuazione della storia e suggerendo una possibile soluzione del problema, mette dunque in gioco una competenza complessa, basata su tutta una serie di competenze parziali (ricettive, produttive, orali, scritte, interculturali, educative, partecipative), il che dimostra il grande potenziale rappresentato dal film nella didattica delle lingue straniere.

\section{Un altro modo di raccontare in didattica}

Anche se nella didattica delle lingue già da tempo si parla della competenza audiovisiva riconoscendola come "quinta abilità" (SCHWERDTFEGER, 1989) ${ }^{16}$, la pratica didattica continua a marginalizzare il film, a ignorare il potenziale cognitivo rappresentato dalla connessione tra parole e immagini, oltre che il potenziale (inter)culturale evidenziato dall'esempio da noi riportato.

Eppure da un lato gli studi neurologici insegnano che la fissazione delle informazioni passa per lo più dal canale visivo (tra l'80 e il 90\%) e che invece viene memorizzato solo il $13 \%$ delle informazioni veicolate dal canale uditivo. La comprensione di quello che si vede insieme a quello che si sente - resa possibile dal film in modo autentico - assume un significato insostituibile per lo sviluppo della competenza produttiva orale. Per la sua caratteristica mimetica il film dall'altro presenta immagini, situazioni, conflitti che assomigliano a quelli della vita

$15 \mathrm{Sul}$ piano didattico è importante che le situazioni tematizzate in classe si colleghino col mondo e le esperienze degli apprendenti. Come scrive Lothar Bredella (2008, p. 15-16): "It is important for the teaching of literature that we encourage students to direct their attention to the question of how the literary text affects them. This includes that they relate the world of the literary text to their own" ("Ė importante nell'insegnamento della letteratura incoraggiare gli studenti a chiedersi come il testo letterario li colpisca. Ciò include il mettere in collegamento il mondo del testo letterario con il proprio mondo").

16 Colpisce che già più di venti anni fa Inge Christine Schwerdtfeger parlasse dell'enorme potenziale del film nell'insegnamento delle lingue, riconoscendo la competenza visiva come "la quinta abilità". 
reale. I materiali tradizionali per l'insegnamento delle lingue presentano invece personaggi "paralizzati”, situazioni asettiche, ambienti fissi e privi di rumori, glossari privi di espressioni idiomatiche in contesto, in cui mancano i vocaboli per descrivere gesti, mimica, linguaggio del corpo: un deficit che l'impiego del film offre la possibilità di colmare (Ibid.). La specifica caratteristica mimetica del film, che come nessun altro mezzo di comunicazione riesce a creare l'impressione di somiglianza con la realtà (WINTERSTEINER, 2003), grazie al confronto con situazioni conflittuali e modi di vedere diversi può dare un importante contributo all'ampliamento della prospettiva individuale, al potenziamento della capacità di percepire se stessi e gli altri (HENSELER; MÖLLER; SURKAMP, 2011), alla sensibilità interculturale. Per questo continua a stupire che il film fino ad oggi non sia inserito in modo sistematico nel sistema scolastico e nella programmazione didattica:

La sua "essenza" non viene elaborata né esercitata a scuola né ci sono istituzioni che lo inseriscano in modo metodico e sistematico come "elemento costitutivo della cultura legittima" (BOURDIEU apud WINTER, 1992, p. 9). ${ }^{17}$

Il compito di immaginare la continuazione della storia crea un ponte con la situazione dell'Europa contemporanea in cui l'arrivo di migliaia di migranti ha cambiato il volto della società, della scuola, della convivenza.
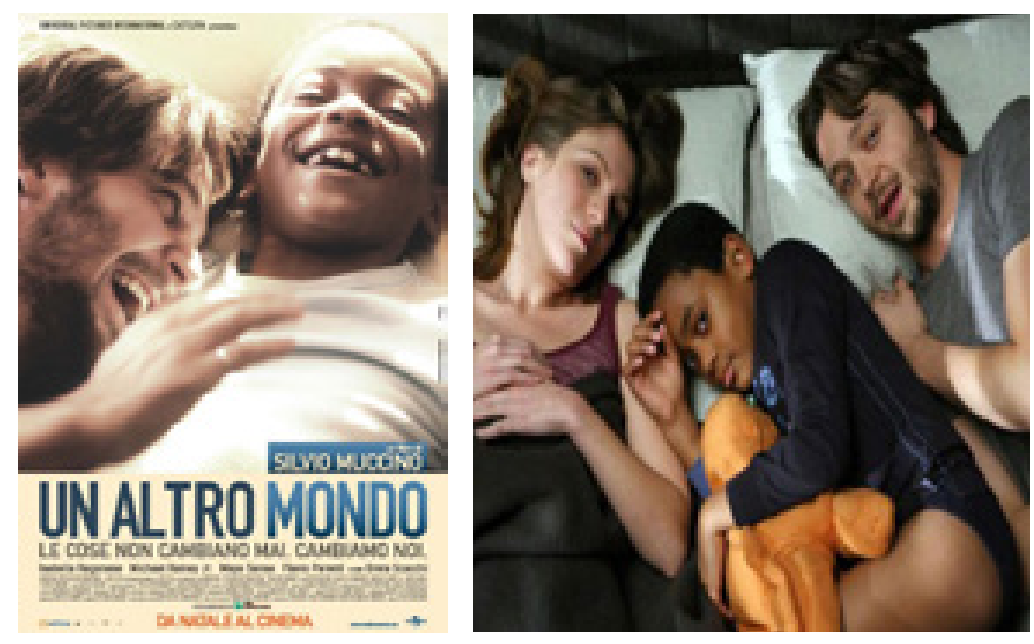

17 „Weder wird sein Wesen in der Schule erarbeitet und eingepaukt, noch gibt es Institutionen, die ihn methodisch und systhematisch als 'konstitutiven Bestandteil der legitimen Kultur' (Bourdieu) ausweisen". 


\subsection{Collegare il mondo del testo al proprio mondo}

"Chi apprende una lingua diventa plurilingue e sviluppa interculturalità" (QCER, 2002, p. 55): il tema della migrazione, che ha rappresentato il fulcro tematico del progetto di collaborazione tra università e scuola, proprio attraverso Un altro mondo ha costituito l'occasione per confrontare gli apprendenti con i problemi della realtà circostante ${ }^{18}$, con i problemi della società e della scuola multiculturale e plurilingue sia in Italia che in Austria. In entrambi i paesi, la dimensione crescente dei flussi migratori ha posto e continua a porre il problema dell'integrazione degli immigrati; sia in Italia che in Austria la scuola, una delle prime e più importanti istituzioni con cui i migranti entrano quasi subito in un rapporto stabile, è veicolo di integrazione attraverso il riconoscimento della scelta dell'educazione interculturale. Come recita un documento ministeriale del febbraio 2014:

una molteplicità di lingue e di culture sono entrate nella scuola. L'intercultura è già oggi il modello che permette a tutti i bambini e ragazzi il riconoscimento reciproco dell'identità di ciascuno. A centocinquanta anni dall'Unità l'italiano è diventata la lingua comune di chi nasce e cresce in Italia (p. 4).

Il tema della migrazione è un tema strutturale della storia italiana a partire dall'Unità: gli italiani emigrati tra il 1870 e il 1980 furono complessivamente 27 milioni, tanti quanti ne contava la penisola al momento dell'unificazione politica del 1861. Centocinquanta anni dopo, la migrazione continua a essere un fenomeno strutturale della storia contemporanea che ha assunto un grande rilievo mediatico da quando, a partire dagli anni Novanta, migliaia di emigranti nordafricani ${ }^{19}$ cercano quasi quotidianamente di approdare sulle coste italiane: negli ultimi 20 anni sono almeno 19.812 le persone morte lungo le frontiere dell'Europa, inghiottite dai flutti

18 "The teaching of literature has to highlight the readers' personal and emotional involvement with the text and how they relate the world of the literary text to their own" ("L'insegnamento della letteratura deve illustrare il coinvolgimento personale ed emozionale dei lettori con il testo e il modo in cui mettono in collegamento il mondo del testo letterario con il proprio") (BREDELLA, 2008, p. 14).

19 Negli anni '90 hanno cominciato a arrivare via mare migranti dal nord dell'Africa; a partire dal 2010 è aumentato il numero dei migranti provenienti dall'Europa centro-orientale (sia UE che non UE) via terra, tanto che oggi la prima comunità straniera è rappresentata dai rumeni (circa un milione di residenti - oltre un quinto di tutti gli stranieri residenti - secondo i dati ISTAT del 1 gennaio 2011). Oltre ai rumeni si registra il $24 \%$ di migranti provenienti dalla Moldavia, il 18,3\% dalla Federazione Russa, il 15,3\% dall'Ucraina, 1'11,1\% dalla Bulgaria. Incrementi notevoli anche da parte di cittadini provenienti dai paesi del sud-est asiatico: Pakistan $(+16,7 \%)$, India (+14,3\%), Bangladesh $(+11,5 \%)$, Filippine $(+8,65)$, Sri Lanka $(+7,6 \%)$. Si vedano le statistiche ISTAT: http://ssai.interno.it/download/ allegatil/amelio_dati-popolazione_straniera_residente_in_italia_-22_set_2011_-_testo_integrale[1].pdf(ultimo accesso 10.7.2014). Gli stranieri residenti in Italia al primo gennaio 2013 sono 4.387.721, 334.000 in più rispetto all'anno precedente. I nati stranieri nel 2012 costituiscono il 15\% del totale dei nati da residenti in Italia (si veda file: http://www.istat.it/it/archivio/96694, ultimo accesso 10.7.2014). 
mentre "tentavano di espugnare la fortezza Europa"20.

Il personaggio di Charlie, la nascita del suo rapporto col fratello ricco e viziato, il cambiamento apportato nella vita di entrambi da questo incontro ravvicinato e inaspettato hanno creato negli studenti occasioni di discussione e fornito preziosi input di riflessione interculturale, hanno suscitato compiti volti a cercare soluzioni a problemi interculturali servendosi della capacità discorsiva in lingua straniera (HALLET, 2012).

\subsection{Un percorso interculturale tra società e scuola}

In un approccio didattico basato sui compiti, al cui centro stanno gli apprendenti in qualità di persone in grado di negoziare significati anche in situazioni complesse (DELANOY, 2004), si è continuato a lavorare con temi e input del film proponendo dei compiti a partire dalle tracce 9 ("Il tema") e 11 ("Novità").

Andrea, colpito dall'ingenuità di Charlie e dal suo bisogno di vicinanza, dopo un viaggio in Kenia costellato di (dis)avventure che hanno creato complicità fraterna, decide suo malgrado di portarlo a Roma e di offrirgli asilo nella propria grande casa in attesa di trovare una famiglia disposta ad adottarlo.

Nella terza parte del film ${ }^{21}$, quella della nuova vita romana dei due "fratelli per caso", Andrea si scontra con gli atteggiamenti xenofobi del mondo della scuola (1:08: 21-1:12: 57). A Charlie, che sostiene di non volerci più andare perché "I compagni mi odiano. Nessuno mi parla e Giovanni mi chiama sempre «cacca nera»", Andrea ribatte: "Charlie, non va bene, tu gli devi rispondere a Giovanni, non puoi permettergli di farti mettere sotto in questo modo. La prossima volta che lui ti offende, tu digli «cacca bianca»".

Poi Andrea si scontra con il razzismo, la superficialità e l'indifferenza di quelli che fino all'arrivo di Charlie credeva suoi amici (1:32:59-1:35:49) e li vede come ragazzi viziati e viziosi. Al ritmo accattivante della canzone $I$ Watussi, di fronte al comportamento xenofobo e discriminante di Tommaso nei confronti di Charlie, Andrea si sente estraneo al mondo dissoluto, fatto di soldi, droga, festini e alcool in cui fino a poco prima trascorreva le giornate e scopre il sentimento di fratellanza e la partecipazione al destino degli esclusi.

Ecco una scelta di compiti orientati all'azione e alla produzione (SURKAMP, 2007) oltre che alla partecipazione e alla riflessione interculturale, proposti nelle classi ginnasiali e agli

20 Dati tratti dal blog Fortress Europe che li raccoglie analizzando le notizie riportate dalla stampa internazionale degli ultimi 23 anni: Un cimitero chiamato Mediterraneo (http://fortresseurope.blogspot.co.at))

21 Il vero difetto del film è probabilmente l'eccessiva lunghezza, il tentativo ambizioso di raccontare una storia di formazione scandita da temi scottanti, in cui sono entrati troppi cliché. Va ricordato che, dopo Un altro mondo, Muccino e la Vangelista si sono impegnati nella Onlus World Friends con la campagna «Nati nel posto giusto» finanziata con sms per sostenere il reparto di maternità del Neema Hospital di Nairobi, oltre che con gli introiti della vendite della favola Fishandchips scritta da Vangelista. La favola si ispira al dinosauro di pezza, da cui Charlie e il giovane attore che lo ha rappresentato non si staccano mai. 
studenti universitari:

\section{Compiti orientati all'azione e alla produzione Compiti orientati alla partecipazione e alla riflessione interculturale}

Diario di lettura: documentazione delle prime Discussione: come reagireste se un ragazzo di colore impressioni dopo la visione delle sequenze del film. venisse escluso e discriminato nella vostra classe?

Formulare una lettera, la pagina di un diario, una mail Scrivere un decalogo di consigli sull'accoglienza e dal punto di vista di Livia, di Andrea o di Charlie sul l'inclusione, di cui far uso in una classe plurilingue difficile inserimento scolastico del bambino. e multiculturale.

Riformulare la scenografia della traccia 9. La voce fuori campo di Charlie commenta lo scontro tra lui e i suoi compagni di classe che lo hanno escluso e offeso.

Prendete a modello la scenografia della traccia 1 , in cui la voce fuori campo di Andrea introduce la storia. Riscrivete la traccia 11 usando la voce fuori campo di Andrea per commentare il comportamento razzista e xenofobo del suo amico Tommaso.

Immaginare un finale alternativo del film dopo Mettere in collegamento quanto visto e ascoltato lo scontro tra Andrea e il suo amico Tommaso, con situazioni viste e ascoltate nel proprio mondo colpevole di aver fatto ubriacare Charlie e di essersi (p.e. sulla discriminazione negli stadi di calcio preso gioco di lui. italiani nei confronti dei calciatori di colore).

Scrivere e mettere in scena un dialogo tra Andrea e Tommaso.

Cercare articoli di cronaca sull'integrazione dei ragazzi stranieri nel sistema scolastico e sociale del proprio paese.

Mettere in scena il conflitto a scuola tra i bambini e Charlie.

Preparare una documentazione sotto forma di rivista letteraria sul tema "Integrazione e educazione interculturale".

\section{Un romanzo multimodale: tra lingua e cultura}

Febbre gialla avrebbe dovuto essere il terzo romanzo di Lucarelli con i protagonisti Coliandro (il poliziotto presuntuoso, imbranato e pasticcione) e Nikita (la sua pallida e giovane protetta) ${ }^{22}$ e sarebbe dovuto uscire per la casa editrice Granata Press che invece fallì. Dopo qualche anno Coliandro divenne Vittorio, poliziotto con la febbre al primo giorno di lavoro alla Squadra

22 Coliandro fece il suo esordio letterario come ispettore della questura di Bologna nel 1991 col breve racconto Nikita della raccolta I delitti del Guppo 13, a cui nel 1993 seguì Falange Armata. Nel 1994 alcune storie sceneggiate da Lucarelli e illustrate da Onofrio Catacchi servirono a realizzare un fumetto basato su Coliandro che, nel 2003 divenne il protagonista della serie televisiva L'Ispettore Coliandro, prodotta fino al 2010. Ispirato ai romanzi di Lucarelli, ogni episodio è stato però un film a se stante, ogni volta con un modello ispiratore diverso (p.e. i film interpretati negli anni Settanta e Ottanta da Tomás Milían nel ruolo dell'Ispettore Nico Giraldi, oppure i polizieschi di Clint Eastwood nel ruolo dell'Ispettore Harry Callaghan). 
Mobile di Bologna, Nikita la sua sorellina Angelika e il giallo venne pubblicato nel 1997 da EL nella collana La casa del giallo. Crimini e delitti ${ }^{23}$. Tenuto conto del titolo enigmatico e della copertina accattivante (da leggere come stimolo visivo), visto che si tratta di un romanzo multimodale - in cui al di là dello sviluppo della fabula, si trovano molte rappresentazioni simboliche e modalità semantiche di tipo non narrativo (HALLET, 2008), come frasi in scritture e stili tipografici diversi, simboli, poesie, pagine in alfabeto diverso da quello romano - Febbre gialla è stato inserito nel progetto didattico nella convinzione che un romanzo multimodale rappresenta una sfida ancora da affrontare nell'insegnamento della letteratura di lingua straniera. Tanto più che il giallo multimodale di Lucarelli ha un tema attuale come il lavoro clandestino e lo sfruttamento dei minori: testi letterari, film, articoli di cronaca collegati in una rete di significati che Clifford Geertz (1983, p. 9) e più tardi Terry Eagleton (2001, p. 51) hanno definito "cultura".

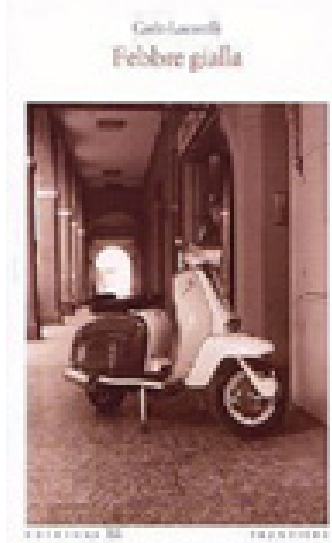

Dopo la lettura della cover del romanzo, che ha suscitato diverse proposte interpretative ${ }^{24}$, si è parlato della comunità cinese in Italia, la quarta dopo quelle romena, albanese e marocchina, che secondo i dati ISTAT al 31 dicembre 2010 contava 209.934 residenti, pari allo 0,34\% del totale della popolazione residente in Italia ${ }^{25}$. Si è chiesto agli studenti di informarsi sulla tragedia del lavoro nero e dell'immigrazione clandestina nelle aziende tessili cinesi della provincia di Prato: molti incidenti, ampiamente riportati dai media, sono avvenuti proprio nel periodo in cui a scuola e all'università si stava lavorando al progetto. Gli studenti universitari si sono informati anche

23 Informazioni ricavate dal sito di Carlo Lucarelli http://www.carlolucarelli.net/index2.htm (ultimo accesso. 18.7.2014).

24 Gli studenti hanno fatto subito delle associazioni con il musical La febbre del sabato sera (Saturday Night Fever del 1977, diretto dal regista John Bendam, con John Travolta); hanno pensato a uno stato patologico dei protagonisti del romanzo; qualcuno poi, valorizzando il suggerimento dell'insegnante relativo alle comunità straniere presenti in Italia, ha fatto associazioni con il colore della pelle della popolazione asiatica.

$25 \mathrm{Si}$ veda http://demo.istat.it/str2010/index.html, Istat, 31 dicembre 2010. URL consultato il 18.7.2014. 
sul romanzo documentario di Edoardo Nesi Storia della mia gente, vincitore del Premio Strega 2011, che tematizza proprio il tracollo della piccola industria tessile pratese, "di imprenditori italiani sbranati dai cinesi senza che nessuno alzi un dito per difenderli" (NESI, 2011, p. 58) ${ }^{26}$. Sono queste le attività didattiche della fase pre-reading.

I while reading e i post reading tasks si sono concentrati per gli studenti ginnasiali sul Prologo di Febbre gialla, narrato da Lucarelli secondo le coordinate della fiaba con chiari richiami intertestuali ${ }^{27}$ e redatto in corsivo come tutte le altre parti del testo in cui la voce del narratore onnisciente dipana la storia di "Hô San-Qwei che si pronunciava Osancuei" (LUCARELLI, 1997, p. 24). Hô, nato " a Soochow, nella provincia di Shangai, il 25 dicembre 1988, sotto il segno del Dragone" (LUCARELLI, loc. cit.), è costretto con altri quindici "seduti l'uno accanto all'altro su quindici sgabelli tutti uguali, davanti a quindici banchi tutti uguali" (Ibid., p. 9) a cucire borse di pelle in una cantina di Bologna, "fermandosi solo ogni tanto per dormire un po' e fare i bisogni in un secchio coperto da un'asse, in fondo alla stanza" (Ibid.,p. 10). Si è lavorato su attività didattiche di vario tipo per verificare in primo luogo la competenza ricettiva e potenziare le strategie di lettura, alla ricerca di spunti per la produzione orale e per la produzione scritta ${ }^{28}$.

Per gli studenti universitari le attività didattiche si sono concentrate, oltre che sulla competenza produttiva orale e scritta e sulla competenza interculturale, sulla sintassi narrativa del romanzo completo e sulle caratteristiche del romanzo multimodale, al fine di esercitare la competenza letteraria in lingua straniera (secondo il modello di SURKAMP, 2012).

Si è ricostruita la vicenda di Vittorio - raccontata in prima persona dalla voce del narratore autoriale -, giovane poliziotto appassionato di musica punk-rock, colpito in pieno, a bordo della sua $2 \mathrm{CV}$, da Hô in canottiera, da "un bambino cinese dalle ginocchia tutte scorticate" (LUCARELLI, 1997, p. 15) a bordo di

una LAMBRETTA, una mitica, meravigliosa, originale LAMBRETTA DEGLI ANNI '60. Bianca, aerodinamica, cromata, lucidisssssssima e completa di specchietti laterali. Il sogno, anzi, il SOOGNO, anzi IL SOOOGNO della vita di ogni vero Scooter Boy (Ibid., p. 12)

\footnotetext{
26 Il testo riportato è in corsivo nell'originale; si tratta della lettera che "Edoardo Nesi, imprenditore a Prato" ha scritto, senza avere il coraggio di spedirla, "a Francesco Giavazzi, economista di punta ed editorialista del 'Corriere della Sera', forse il più acerrimo sostenitore italiano dell'infinita bontà della globalizzazione” (NESI, 2011, p. 53).

27 "I bambini erano quindici, seduti l'uno accanto all'altro su quindici sgabelli tutti uguali, davanti a quindici banchi tutti uguali, rotondi e di legno nero": questo l'incipit del testo, che richiama la favola di Jakob e Wilhelm Grimm Schneewittchen (1857), tradotta in italiano come Biancaneve e i sette nani, in francese come Blancheneige. Da ora in poi le citazioni dal testo di Lucarelli verranno indicate direttamente nel testo.

$28 \mathrm{Si}$ vedano a questo proposito i materiali didattici approntati da studenti universitari del corso di laurea in italiano abilitante all'insegnamento dell'Università di Salisburgo. Questo il link alla piattaforma: www.italianoAscuola.at. Si veda anche: RÜCKL, MORIGGI (2013).
} 
Il bambino, dopo lo scontro, se l'è data a gambe, lasciando il poliziotto in erba con l'interrogativo irrisolto:

COSA CI FA UN BAMBINO CINESE

DALL'APPARENTE ETÀ DI OTTO-DIECI ANNI

SU UNA MERAVIGLIOSA LAMBRETTA STILE

REAL OLD SIXTIES SPARATO A TUTTA

VELOCITÀ SOTTO UN PORTICO? (Ibid., p. 16)

Come si vede, già il primo capitolo del testo, intitolato " $37^{\circ}$ e mezzo" - ognuno dei 7 capitoli porta come titolo il numero dei gradi della temperatura corporea di Vittorio, che nell'ultimo sale a 40 -, suscita curiosità nel lettore proprio per le sue caratteristiche multimodali: nel testo compaiono onomatopee, particolarità grafiche, parole in stampatello, parole in grassetto e poco dopo quasi una pagina di pittogrammi. Si tratta del Passport of Chang Qwahn-Zi che Vittorio si fa tradurre da un collega dell'Ufficio Stranieri, per scoprire che:

I documenti sono veri e intestati a questo Chang, ma la fotografia non corrisponde. Chang Qwahn-Zi è nato a Pengpu, nella provincia di Nanchino, il 15 ottobre del 1872. Complimenti, Vittorio. Ti sei fatto speronare la macchina da un bambino cinese di centoventicinque anni (Ibid., p. 23).

Sullo sfondo di una Bologna fredda e buia, che ignora i traffici illeciti organizzati nelle cantine dei suoi vicoli, Vittorio si butta alla ricerca di quel bambino di centoventicinque anni, e con la febbre a 40 riuscirà a smascherare un' organizzazione criminale cinese che sfrutta il lavoro minorile. Le attività proposte sono servite a potenziare l'abilità di lettura di un romanzo multimodale come Febbre gialla, che si è rivelato molto adatto a una generazione di adolescenti spesso estranei alle forme della narrazione tradizionale, per i quali la combinazione di diversi modi e media può rappresentare invece una sfida e anche una forma di identificazione. Con le varie attività proposte sono state potenziate le seguenti competenze parziali: 


\begin{tabular}{|l|l|l|}
\hline Comprensione estetica: & $\begin{array}{l}\text { Competenza linguistico- } \\
\text { discorsiva: }\end{array}$ & $\begin{array}{l}\text { Competenza interculturale e } \\
\text { riflessiva: }\end{array}$ \\
$\begin{array}{l}\text { riconoscere il procedimento } \\
\text { narrativo }\end{array}$ & $\begin{array}{l}\text { usare le strutture della lingua } \\
\text { individuate nel testo e i suoi } \\
\text { procedimenti narrativi per scrivere } \\
\text { testi analoghi }\end{array}$ & $\begin{array}{l}\text { mettere in collegamento quanto } \\
\text { letto col proprio mondo, con le } \\
\text { proprie esperienze }\end{array}$ \\
$\begin{array}{l}\text { riconoscere le caratteristiche e } \\
\text { le particolarità estetiche di un } \\
\text { testo letterario rispetto ad altri } \\
\text { tipi di testo (rispetto a un testo } \\
\text { referenziale p.e.) }\end{array}$ & $\begin{array}{l}\text { trasformare il testo letto in un altro } \\
\text { tipo testuale }\end{array}$ & partecipazione emotiva \\
$\begin{array}{l}\text { riconoscere e analizzare } \\
\text { le strutture della lingua e i } \\
\text { procedimenti specifici usati }\end{array}$ & $\begin{array}{l}\text { caparti empatia } \\
\text { vista diversi dal proprio }\end{array}$ \\
\hline
\end{tabular}

\section{Conclusioni e prospettive}

Parlare di competenze ci ricorda che dopo la rivoluzione copernicana degli anni '70 in cui il metodo comunicativo ha stravolto la didattica delle lingue puntando l'accento sulle funzioni, a partire dagli anni '90 è stato proprio il concetto di competenza a permeare l'ambito della didattica, spostando la ricerca dalle funzioni della lingua ai processi mentali degli apprendenti, alla misurabilità dei processi di apprendimento stessi. Il che ci riporta anche alla radice etimologica comune del termine competenza e del termine competizione. Questo è il motivo per cui la competenza interculturale, permeata come si è visto da fattori soggettivi, legata a emozioni, sensibilità ed esperienze personali risulta difficilmente conciliabile con la misurabilità delle altre competenze. L'esempio che abbiamo presentato dovrebbe aver dato risposta alla domanda da cui siamo partiti: la competenza culturale e la partecipazione devono avere spazio e un ruolo fondamentale in ogni tipo di insegnamento. È necessario perciò che chi insegna una lingua straniera sia in grado di mettere insieme formati didattici collegati ai discorsi del mondo e riesca a utilizzare il complesso potenziale che i testi letterari rappresentano nella moderna didattica delle lingue perché "solo in riferimento a discorsi sociali reali possono essere create condizioni favorevoli e motivanti di apprendimento, in cui gli apprendenti attribuiscano rilevanza 
e importanza ai contenuti di insegnamento" (HALLET, 2007, p. 33$)^{29}$.

\section{Riferimenti bibliografici}

ARNOLD, M. Aspekte einer modernen Neurodidaktik. Emotionen und Kognitionen im Lernprozess. München: Ernst Vögel, 2002.

BALBONI, P. E. Le sfide di Babele. Insegnare le lingue nelle società complesse. Novara: De Agostini, 2002. . Le sfide di Babele. Insegnare le lingue nelle società complesse. 3. ed. Novara: De Agostini, 2012. . Lo sviluppo delle abilità recettive. In: . Fare educazione linguistica. Insegnare italiano, lingue straniere e lingue classiche. Novara: De Agostini, 2013, pp. 95-116.

BOURDIEU, P. Die gesellschaftliche Definition der Fotografie. In BOURDIEU, P.; L. BOLTANSKI, L. (eds.). Eine illegitime Kunst. Die sozialen Gebrauchsweisen der Fotografie. Frankfurt /M. u.a.: E.V.A. 1981, 85-109.

BREDELLA, L. What makes Reading Literary Texts Pleasurable and Educationally Significant? In: Fremdsprachen Lehren und Lernen, Tübingen: 37, pp.12-26, 2008.

CONSIGLIO D'EUROPA. Quadro Comune Europeo di Riferimento per le lingue: apprendimento insegnamento valutazione. Trad. F. Quartapelle e D. Bertocchi. Firenze: La Nuova Italia-Oxford, 2002.

DELANOY, W. Rezeptionsästhetische und Task-Based-Learning am Beispiel von Meera Syals Roman Anita and Me. In: BREDELLA, L.; DELANOY, W.; SURKAMP, C. (a cura di). Literaturdidaktik im Dialog. Tübingen: Narr, 2004, pp. 147-180.

DIADORI, P. Il cinema per l'insegnamento dell' italiano L2. In: DIADORI, P.; MICHELI, P. Cinema e didattica dell'italiano L2. Perugia: Guerra, 2010, pp. 192-257.

EAGLETON, T. Was ist Kultur. München: dtv, 2001.

ECO, U. Sei passeggiate nei boschi narrativi. Harvard University, Norton Lectures 1992-1993. Milano: Bompiani, 1994.

. Sulla letteratura. Milano: Bompiani, 2002.

FABBRO, F. Il cervello bilingue. Neurolinguistica e poliglossia. Roma: Astrolabio, 1996.

GEERTZ, C. Dichte Beschreibung. Beiträge zum Verstehen kulturelle Systeme. Frankfurt: Suhrkamp, 1983.

HALLET, W. Die komplexe Kompetenzaufgabe. Fremdsprachliche Diskursfähigkeit als kulturelle

29 „Nur durch den Bezug auf reale gesellschaftliche Diskurse können motivierende und günstige Lernbedingungen geschaffen werden, in denen die Lernenden den Unterrichtsinhalten Relevanz und Bedeutsamkeit beimessen " (HALLET, 2007, p. 33). 
Teilhabe und Unterrichtspraxis. In: HALLET, W.; KRÄMER, U. (a cura di). Kompetenzaufgaben im Englischunterricht. Grundlagen und Unterichtsbeispiele. Seelze: Friedrich Verlag, 2012, pp. 8-18.

HALLET, W. Literatur und Kultur im Unterricht: Ein kulturwissenschaftlicher didaktischer Ansatz. In: HALLET, W.; NÜNNING, A. (a cura di). Neue Ansätze und Konzepte der Literatur- und Kulturdidaktik. Trier: WVT, 2007, pp. 31-48.

HALLET, W. Visual culture and Literature: Multimodale Romane, Literaturunterricht und Literaturdidaktik. In: Fremdsprachen Lehren und Lernen, [Tübingen]: 37, pp. 141-153, 2008.

HALLET, W.; NÜNNING, A. (a cura di). Romandidaktik. Theoretische Grundlagen, Methoden, Lektüreanregungen. Trier: WVT, 2009.

HENSELER, R.; MÖLLER, S.; SURKAMP, C. (a cura di). Filme im Englischunterricht. Grundlagen, Methoden, Genres. Seelze: Kallmeyer-Klett, 2011.

ISSING, L.; HANNEMANN, J. (a cura di). Lernen mit Bildern. Grünewald: Institut für Film und Bild im Wissenschaft und Unterricht, 1983.

ITALIA. Ministero dell'Istruzione, dell'Università e della Ricerca. Linee guida per l'accoglienza e l'integrazione degli alunni stranieri. 2014, http://www.istruzione.it/allegati/2014/linee_guida_ integrazione_alunni_stranieri.pdf Acesso: 10.7.2014

LUCARELLI, C. Falange armata. Bologna: Metrolibro, 1993. . Febbre gialla. Trieste: EL, 1997. . Nikita, Bologna: Metrolibro, 1991. L'ispettore Coliandro. Torino: Einaudi, 2009.

MUCCINO, S. Un altro mondo, con Silvio Muccino, Isabella Ragonese, Michael Rainey Jr, Maya Sansa, Italia, 109', 2010.

NESI, E. Storie della mia gente. Milano: Bompiani, 2011.

RAINER, W. Filmsoziologie. Eine Einführung in das Verhältnis von Film, Kultur und Gesellschaft. München: Quintessenz, 1991.

RÜCKL, M.;, MORIGGI, R. I mondi di Nikita e Vittorio: due racconti gialli di Carlo Lucarelli nella classe di italiano. In: RÜCKL, M.; SANTORO, E.; VEDDER, I. (a cura di). Contesti di apprendimento di italiano L2. Tra teoria e pratica didattica. Roma: Franco Cesati, 2013, p. 129-140.

SARTRE, J. P. Qu'est-ce que la literature? Paris: Gallimard, 1964, p. 58-93.

SCHWERDTFEGER, I. C. Sehen und Verstehen. Arbeit mit Filmen im Unterricht Deutsch als Fremdsprache, Berlin: Langenscheidt, 1989.

SURKAMP, C. Literarische Texte im kompetenzorientierten Fremdsprachenunterricht. In: HALLET, 
W.; KRÄMER, U. Kompetenzaufgaben im Englischunterricht Grundlagen und Unterrichtsbeispiele. Seelze: Kallmayer, 2012.

SURKAMP, C. Handlungs- und Produktionsorientierung im fremdsprachlichen Literaturunterricht. In: HALLET, W.; NÜNNING A. Neue Ansätze und Konzepte der Literatur- und Kulturdidaktik. Trier: WVT, 2007.

VANGELISTA, C. Un altro mondo. Milano: Feltrinelli, 2009.

WINTER, R. Filmsoziologie. Eine Einführung in das Verhältnis von Film, Kultur und Gesellschaft. München: Quintessenz, 1992.

WINTERSTEINER, W. Eine andere Art zu erzählen. In: ide. die zeit-schrift für den deutschunterricht in wissenschaft und schule, 4-2003, pp. 8-15.

\section{Sitografia}

http://www.socialstudies.org/positions/medialiteracy (ultima consultazione 7.7.2014).

http://cinema.ilsole24ore.com/film-brevi/2010-12-23/cose-dellaltro-mondo-00017792. php? uuid=AYJgs8tC (ultimo accesso 5.7.2014).

http://www.film.it/recensione/art/un-altro-mondo-la-nostra-recensione-7935/ (ultimo accesso 15.7.2014)

www.carlavangelista.it/ (ultimo accesso 10.7.2014).

http://www.ilsole24ore.com/art/cultura/2010-12-28/silvio-muccino-cose-altro-130254.shtml\# (ultimo accesso 16.7.2014).

http://www.istruzione.it/allegati/2014/linee_guida_integrazione_alunni_stranieri.pdf(ultimo accesso 10.7.2014)

http://ssai.interno.it/download/allegatil/amelio_dati-popolazione_straniera_residente_in_italia_-_22_ set_2011_-_testo_integrale[1].pdf(ultimo accesso 10.7.2014)

http://fortresseurope.blogspot.co.at/ (ultimo accesso 10.7.2014)

http://www.carlolucarelli.net/index2.htm (ultimo accesso. 18.7.2014) 